\section{Current status of high-intensity focused ultrasound for the management of uterine adenomyosis}

\author{
Vincent Y. T. Cheung \\ Department of Obstetrics and Gynaecology, Queen Mary Hospital, The University of Hong \\ Kong, Hong Kong
}

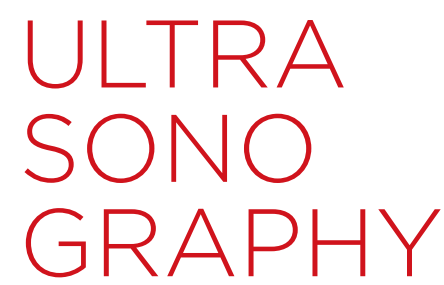

https://doi.org/10.14366/usg. 16040 pISSN: 2288-5919 e eISSN: 2288-5943 Ultrasonography 2017;36:95-102

Received: October 1, 2016

Revised: November 27, 2016 Accepted: November 30, 2016

Correspondence to: Vincent Y. T. Cheung, MBBS, FRCOG, FRCSC, Department of Obstetrics and Gynaecology, Queen Mary Hospital, The University of Hong Kong, 102 Pokfulam Road, Hong Kong

Keywords: Adenomyosis; High-intensity focused ultrasound; Ultrasonography

\title{
Introduction
}

Uterine adenomyosis is a common gynecologic disorder of women of reproductive age. It is characterized by the presence of ectopic endometrial glands and stroma within the myometrium, resulting in uterine enlargement. It can be asymptomatic, but can give rise to significant symptoms, most commonly menorrhagia and dysmenorrhea [1]. The treatment for adenomyosis can be challenging. Historically, the standard of treatment has been hysterectomy. However, this is not always an acceptable option, especially for women who wish to maintain their fertility. Medical treatment mainly allows the control of symptoms, whereas uterine-sparing surgical techniques, such as the excision of adenomyotic foci or electrocoagulation of the involved myometrium, are associated with variable success and risk of recurrence $[1,2]$. Although uterine artery embolization has been used as a minimally invasive treatment option with some success, its impact on future fertility and pregnancy remains uncertain $[1,3,4]$.

Recently, increasing attention has been paid to high-intensity focused ultrasound (HIFU) therapy for the management of adenomyosis [5,6]. It induces focal thermocoagulation of the adenomyotic lesions and has been shown to be a safe and effective treatment for adenomyosis $[5,6]$. Magnetic resonance (MR) imaging and, more recently, ultrasound (US) have been used to target and monitor the ablation process $[5,7,8]$. HIFU may also be able to provide an additional minimally invasive treatment option for premenopausal women with uterine adenomyosis. The purpose of this article was to review the background, clinical use, and treatment outcomes of HIFU in the treatment of adenomyosis.

This is an Open Access article distributed under the terms of the Creative Commons Attribution NonCommercial License (http://creativecommons.org/ licenses/by-nc/3.0/) which permits unrestricted noncommercial use, distribution, and reproduction in any medium, provided the original work is properly cited.

Copyright (C) 2017 Korean Society of Ultrasound in Medicine (KSUM)

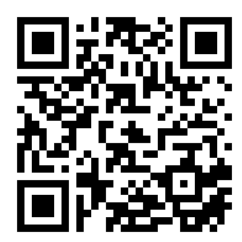

How to cite this article:

Cheung VYT. Current status of high-intensity focused ultrasound for the management of uterine adenomyosis. Ultrasonography. 2017 Apr;36(2):95-102. 


\section{Imaging-Guided HIFU}

In 1942, Lynn et al. [9] introduced the use of an extracorporeal source of focused US energy to induce coagulative necrosis in targeted tissue without damaging surrounding and overlying vital structures. The principle of this HIFU therapy is to cause tissue ablation through heating, cavitation, and direct damage to tumor blood vessels, by focusing US energy at a targeted spot, with minimal damage to the surrounding tissues.

HIFU can be performed under the guidance of MR or US imaging in order to target and monitor the ablation process. MR imaging offers excellent anatomic resolution and temperature sensitivity during real-time treatment monitoring. The ExAblate device (Insightec, Tirat Carmel, Israel) is currently the only United States Food and Drug Administration (FDA)-approved MR-guided HIFU system. Another system, the Sonalleve (Philips Healthcare, Andover, MA, USA), has received Conformité Européene (CE) marking for fibroid treatment.

US-guided HIFU uses grayscale or echogenicity changes to determine the adequacy of ablation instead of temperaturemapping changes, as in MR-guided HIFU. The JC HIFU system (Chongqing Haifu Technology, Chongqing, China) (Fig. 1) has been installed at Queen Mary Hospital since 2006, mainly for the treatment of hepatocellular carcinoma [10]; since 2011, the ablation of symptomatic uterine fibroids has also been performed $[7,8]$. As described in a previous review of fibroid management [7], this HIFU system consists of a real-time 3.5-MHz diagnostic US scanner integrated into the center of a $12-\mathrm{cm}$ in diameter, $15-\mathrm{cm}$ in focal length, 0.8-MHz therapeutic US transducer (Fig. 2). In addition to the US system, it contains a 6-direction therapeutic planning system,

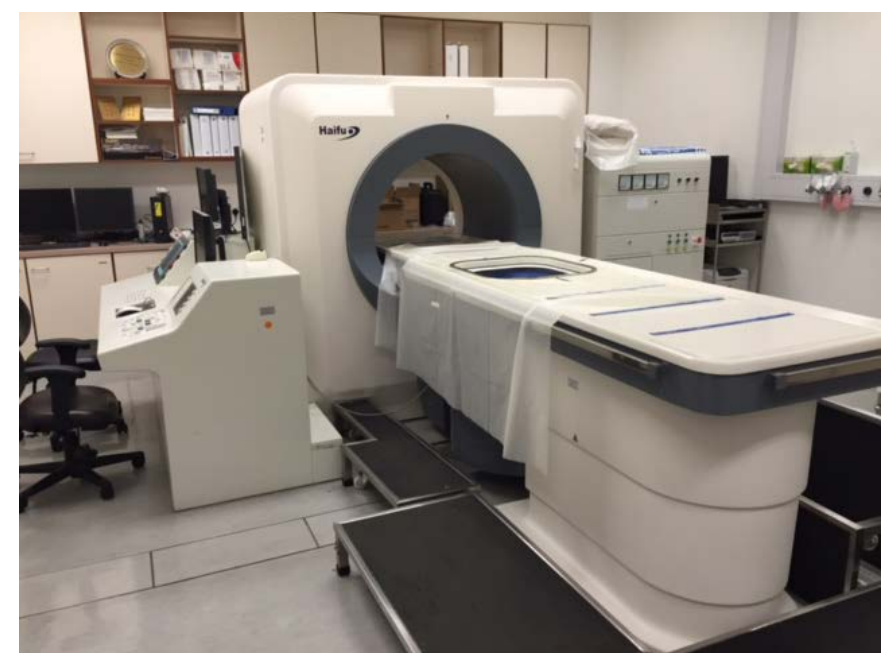

Fig. 1. Ultrasound-guided high-intensity focused ultrasound system. a degassed water circulation system, and a master control computer unit. The system can attain an acoustic output power of up to $400 \mathrm{~W}$, and the linear motion deviation of the therapeutic device is $\pm 1 \mathrm{~mm}$. During treatment, patients are placed in the prone position under intravenous conscious sedation. This enables minimal patient movement while the lower abdominal skin is in contact with the degassed water. The entirety of the lesion is divided into slices of $5 \mathrm{~mm}$. The acoustic output power is set between 350 and $400 \mathrm{~W}$, and with successive sweeps from the deep to the shallow region, the entire volume of the lesion is ablated [7]. Fig. 3 illustrates the MR images of a woman with extensive adenomyosis before and 3 months after HIFU treatment. Other similar systems, including the HIFU-2001 (SJTU Suntec Industry, Shanghai, China), HIFUNIT9000 (Shanghai A\&S Science and Technology, Shanghai, China), and FEPBY Series (China Medical Technologies, Beijing, China) are also currently used clinically for the treatment of fibroids. However, none of these systems described have received FDA approval.

The selection criteria for HIFU therapy for adenomyosis vary depending on the experience of an individual center. The criteria used in most studies can be summarized as follows: (1) premenopausal women of over 18 years of age with no plans for future childbearing [11-16]; (2) significant symptoms related to adenomyosis [11-17]; (3) features of adenomyosis on MR imaging [11,13-16]; (4) adenomyotic lesions greater than $3 \mathrm{~cm}$ but less than $10 \mathrm{~cm}$ in diameter [11,14]; (5) no evidence of known or suspected extensive pelvic adhesions, such as a history of acute pelvic inflammatory disease, severe pelvic endometriosis, or lower abdominal surgery [12-18]; and (6) body weight of less than $100 \mathrm{~kg}$ [16] or abdominal wall thickness of less than $5 \mathrm{~cm}$ [17].

Treatment centers vary in their protocols for patient assessment

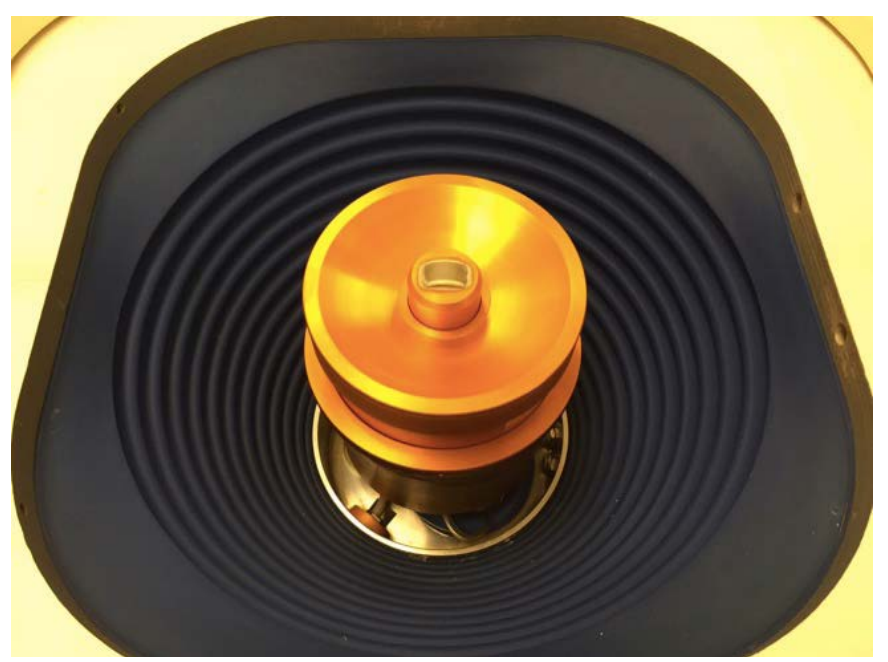

Fig. 2. Real-time diagnostic ultrasound scanner integrated into the center of the therapeutic ultrasound transducer. 


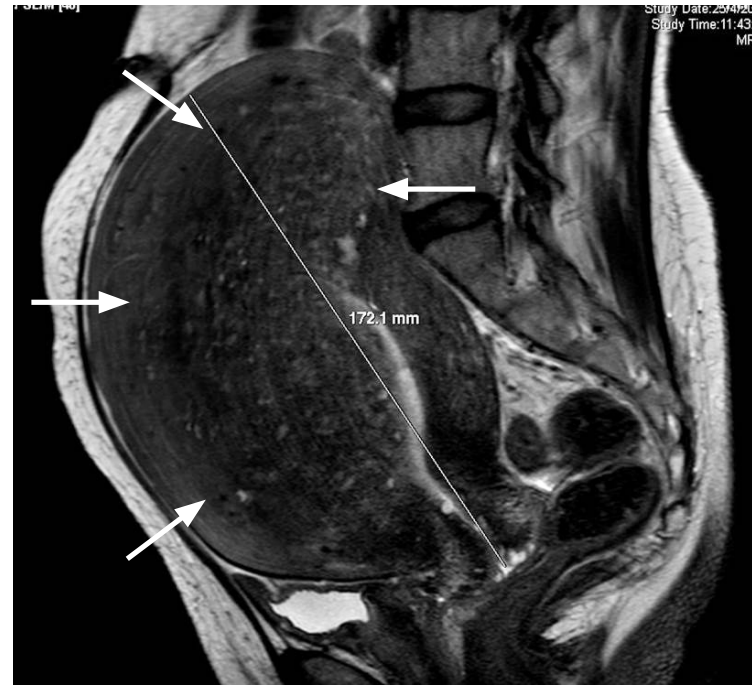

A

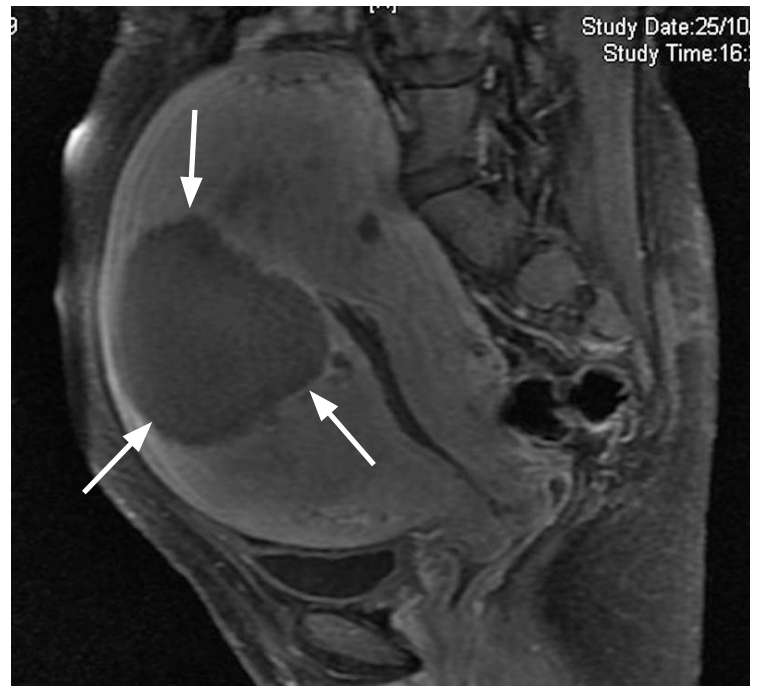

B

Fig. 3. Magnetic resonance (MR) images from a 47-year-old woman with adenomyosis.

A. Pretreatment MR image shows extensive adenomyosis involving mostly the fundal and anterior uterine wall (arrows). B. MR image (with contrast) 3 months after treatment shows a well-defined hypoperfused area (arrows) as the result of focused ultrasound ablation.

and preparation. Pretreatment imaging to confirm the diagnosis and to establish the extent of adenomyosis is performed. Additionally, pretreatment planning is carried out with the patient lying prone on the treatment table, which mimics the treatment process and familiarizes the patient with the treatment. During the planning process, the path of sonication, depth of the target, proximity of the target to the sacrum, and the likelihood of the presence of a bowel loop along the path of sonication are evaluated [7].

\section{Literature Review}

A review of the literature published in English was obtained from the PubMed electronic database from inception through March 2016, using the search words "adenomyosis" and "high-intensity focused ultrasound," "HIFU," or "focused ultrasound ablation," supplemented by hand-searching the references of the relevant articles. The acceptability of the articles to be included in the review was decided by reading the articles' abstracts and full text if necessary, and only those articles that evaluated the use of HIFU in the treatment of adenomyosis without associated fibroids and included complete interpretable information with relevant outcome data were reviewed. Ultimately, 11 articles were reviewed. The information that was retrieved from the eligible articles included the number of patients, treatment details, criteria for symptom assessment, volume of uteri and adenomyotic lesions, and related complications.

Eleven articles were identified, including information from 1,150 treatments and follow-up data from 990 patients [11-21]. Table 1 summarizes the background information of each article. Of these 11 articles, five were MR-guided and six were US-guided HIFU studies, reporting a total of 84 and 1,066 treatments with follow-up data, respectively. Two articles were excluded because their reported treatment(s) were likely duplicates of those reported in other articles $[22,23]$. Among the reviewed articles, seven were prospective cohort studies $[11,12,14-16,18,21]$, three were retrospective reviews $[13,17,20]$, and one was a case report [19]. All articles reported the degree of symptom improvement, but only four reported the degree of uterine volume or adenomyotic lesion reduction $[11,12,14,17]$.

\section{Treatment Outcomes}

Table 2 presents details of the 10 studies that evaluated the improvement of menorrhagia; seven studies used the symptom severity score of a quality of life questionnaire specific to fibroids known as the Uterine Fibroid Symptom and Quality of Life questionnaire [24]; while three studies used the menstrual volume or menorrhagia scale, which was scored according to patients' descriptions on a 5 -point scale $[15,21]$ or as described by Sharp et al. in 1995 [25]. The reported degree of menorrhagia reduction ranged from $12.4 \%$ to $33.3 \%, 25.3 \%$ to $80.8 \%, 16.4 \%$ to $52.4 \%$, $24.9 \%$ to $66.4 \%, 44.0 \%$, and $44.8 \%$ at $1-, 3-, 6-, 12-, 18-$, and 24-month follow-ups, respectively (Table 2).

The reduction of dysmenorrhea, as determined based on the menstrual pain score using a visual analog scale, was evaluated in 
seven studies (Table 3); all showed a reduction of dysmenorrhea at 3 months (range, $25.0 \%$ to $83.3 \%$ ), 6 months (range, $44.7 \%$ to $100 \%$ ), 12 months (range, $64.0 \%$ to $72.1 \%$ ), 18 months $(54.2 \%)$, and 24 months (56.0\%).

Five studies reported the degree of uterine volume reduction after HIFU therapy, with values ranging from $12.7 \%$ to $54.0 \%$ over follow-up periods of 1 to 12 months (Table 4). However, only two articles reported the degree of reduction in adenomyotic lesions over 12 months (Table 4). The nonperfused volume, which is the percentage of the uterine volume ablated and shown as a non- enhancing area on contrast-enhanced T1-weighed MR imaging after the treatment, has been suggested to be associated with the degree of subsequent symptom improvement. This indicator was reported in seven studies, with mean values ranging from $24.4 \%$ to $62.5 \%$ $[11-16,19]$. However, a study that reported a range of $5 \%$ to $99 \%$ was excluded [21].

\section{Treatment Data}

Table 5 shows the treatment data, including treatment and

Table 1. Summary of studies of high-intensity focused ultrasound for adenomyosis

\begin{tabular}{|c|c|c|c|c|c|}
\hline Study & City, country & $\begin{array}{l}\text { High-intensity focused } \\
\text { ultrasound system }\end{array}$ & No. of treatments & $\begin{array}{l}\text { No. of patients who } \\
\text { completed follow-up }\end{array}$ & $\begin{array}{c}\text { Duration of } \\
\text { follow-up (mo) }\end{array}$ \\
\hline \multicolumn{6}{|l|}{ Magnetic resonance-guided } \\
\hline Ferrari et al. [18], 2016 & L'Aquila, Italy & ExAblate $e^{a)}$ & 18 & 18 & 12 \\
\hline Fan et al. [11], 2012 & Chongqing, China & $J M 15100^{b)}$ & 10 & 10 & 12 \\
\hline Polina et al. [19], 2012 & Andhra Pradesh, India & ExAblate $e^{\text {a) }}$ & 1 & 1 & 6 \\
\hline Kim et al. [20], 2011 & Gyeonggi-do, South Korea & ExAblate $^{\text {a) }}$ & 35 & 35 & 6 \\
\hline Fukunishi et al. [12], 2008 & Kobe, Japan & ExAblate $^{\mathrm{a})}$ & 20 & 20 & 6 \\
\hline \multicolumn{6}{|l|}{ Ultrasound-guided } \\
\hline Liu et al. [13], 2016 & Beijing, China & $J C^{b)}$ & 230 & 208 & 3 \\
\hline Lee et al. [17], 2015 & Incheon, South Korea & $J C^{b)}$ & 346 & 346 & 12 \\
\hline Shui et al. [21], 2015 & Chongqing and Sichuan, China & $J\left(200^{b)}\right.$ & 350 & 224 & 24 \\
\hline Long et al. [14], 2015 & Chongqing, China & $J\left(200^{b)}\right.$ & 51 & 47 & 12 \\
\hline Zhou et al. [15], 2011 & Chongqing, China & $J C^{b)}$ & 77 & 69 & 18 \\
\hline Wang et al. [16], 2009 & Beijing, China & $J C^{b)}$ & 12 & 12 & 3 \\
\hline
\end{tabular}

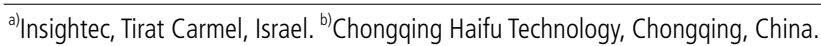

Table 2. Improvement of menorrhagia after high-intensity focused ultrasound for adenomyosis

\begin{tabular}{|c|c|c|c|c|c|c|}
\hline \multirow{2}{*}{ Study } & \multicolumn{6}{|c|}{ Reduction in menorrhagia (\%) } \\
\hline & 1 & 3 & 6 & 12 & 18 & 24 \\
\hline \multicolumn{7}{|l|}{ Magnetic resonance-guided } \\
\hline Ferrari et al. [18], 2016 & - & - & - & 66.4 & - & - \\
\hline Fan et al. [11], 2012 & 12.4 & 25.3 & 16.4 & 24.9 & - & - \\
\hline Polina et al. [19], 2012 & - & 31.6 & 47.4 & - & - & - \\
\hline Kim et al. [20], 2011 & - & 25.9 & 40.7 & - & - & - \\
\hline Fukunishi et al. [12], 2008 & 33.3 & 53.5 & 44.8 & - & - & - \\
\hline \multicolumn{7}{|l|}{ Ultrasound-guided } \\
\hline Lee et al. [17], 2015 & - & 55.6 & 52.4 & 58 & - & - \\
\hline Shui et al. [21], 2015 & - & $44.8^{\mathrm{b})}$ & - & $48.3^{b)}$ & - & $44.8^{b)}$ \\
\hline Long et al. [14], 2015 & - & 32.9 & 50.4 & 64.7 & - & - \\
\hline Zhou et al. [15], 2011 & - & $48.0^{b)}$ & - & - & $44.0^{b)}$ & - \\
\hline Wang et al. [16], 2009 & - & $57.0-80.8^{b, c)}$ & - & - & & - \\
\hline
\end{tabular}

${ }^{a}$ Months after treatment, based on the symptom severity score of the Uterine Fibroid Symptom and Quality of Life questionnaire, unless otherwise specified. ${ }^{b)}$ Based on menstrual volume/menorrhagia score. ${ }^{c}$ Varied depending on power output (290-420 W). 
Table 3. Improvement of dysmenorrhea after high-intensity focused ultrasound for adenomyosis

\begin{tabular}{|c|c|c|c|c|c|}
\hline \multirow{2}{*}{ Study } & \multicolumn{5}{|c|}{ Reduction in dysmenorrhea $(\%)^{a)}$} \\
\hline & 3 & 6 & 12 & 18 & 24 \\
\hline \multicolumn{6}{|c|}{ Magnetic resonance-guided } \\
\hline Polina et al. [19], 2012 & 60 & 100 & - & - & - \\
\hline Kim et al. [20], 2011 & 30.6 & 44.7 & - & - & - \\
\hline \multicolumn{6}{|l|}{ Ultrasound-guided } \\
\hline Liu et al. [13], 2016 & 61.8 & - & - & - & - \\
\hline Shui et al. [21], 2015 & 62 & - & 64 & - & 56 \\
\hline Long et al. [14], 2015 & 28.8 & 53.5 & 72.1 & - & - \\
\hline Zhou et al. [15], 2011 & 56.3 & - & - & 54.2 & - \\
\hline Wang et al. [16], 2009 & $25.0-83.3^{b)}$ & - & - & - & - \\
\hline
\end{tabular}

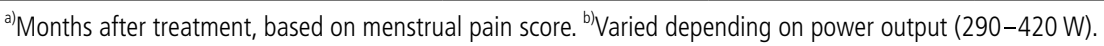

Table 4. Volume reduction of the uterus or adenomyotic lesion after high-intensity focused ultrasound

\begin{tabular}{|c|c|c|c|c|}
\hline \multirow{2}{*}{ Study } & \multicolumn{4}{|c|}{ Reduction in uterine volume or adenomyotic lesion volume $(\%)^{a)}$} \\
\hline & 1 & 3 & 6 & 12 \\
\hline \multicolumn{5}{|l|}{ Magnetic resonance-guided } \\
\hline Fan et al. [11], 2012 & $21.9(11.9)^{a)}$ & $22.5(28.3)^{\mathrm{a})}$ & $29.3(40.2)^{a)}$ & $23.8(29.1)^{\mathrm{a}}$ \\
\hline Fukunishi et al. [12], 2008 & - & - & 12.7 & - \\
\hline \multicolumn{5}{|l|}{ Ultrasound-guided } \\
\hline Lee et al. [17], 2015 & - & 44 & 47 & 54 \\
\hline Long et al. [14], 2015 & - & - & - & $22(30.2)^{a)}$ \\
\hline
\end{tabular}

${ }^{\text {a) }}$ Months after treatment.

Table 5. Treatment data

\begin{tabular}{|c|c|c|c|c|c|}
\hline Study & Treatment time $^{a)}$ & Sonication time ${ }^{\text {b) }}$ & Total exposure energy $(\mathrm{J})$ & Volume of uterus $\left(\mathrm{cm}^{3}\right)$ & $\begin{array}{c}\text { Volume of adenomyotic } \\
\text { lesion }\left(\mathrm{cm}^{3}\right)\end{array}$ \\
\hline \multicolumn{6}{|l|}{ Magnetic resonance-guided } \\
\hline Fan et al. [11], 2012 & $\begin{array}{c}114 \pm 48 \\
(42-192)\end{array}$ & $\begin{array}{c}990.5 \pm 480.6 \\
(245.0-1,727.0)\end{array}$ & $299,019.5 \pm 154,636.0$ & $\begin{array}{c}272.0 \pm 99.2 \\
(148.8-440.0)\end{array}$ & $\begin{array}{c}94.9 \pm 54.6 \\
(30.4-208.4)\end{array}$ \\
\hline Polina et al. [19], 2012 & 110 & NA & 108,996 & NA & 91 \\
\hline Kim et al. [20], 2011 & $150 \pm 40$ & NA & NA & $430 \pm 230$ & NA \\
\hline Fukunishi et al. [12], 2008 & $<180$ & NA & $\begin{array}{c}157,745.4^{c)} \\
(69,066.8-491,840.3)\end{array}$ & $\begin{array}{c}445 \pm 296 \\
(95 \% \mathrm{Cl}, 307-584)\end{array}$ & NA \\
\hline \multicolumn{6}{|l|}{ Ultrasound-guided } \\
\hline Liu et al. [13], 2016 & $\begin{array}{c}64^{c)} \\
(\mid \mathrm{QR}, 47-91)\end{array}$ & $\begin{array}{c}1,135^{c)} \\
(I Q R, 769-1,561)\end{array}$ & NA & $\begin{array}{c}274.4 \pm 174.8 \\
(47-1,390)\end{array}$ & $\begin{array}{l}70.7 \pm 33.0 \\
(16-177)\end{array}$ \\
\hline Lee et al. [17], 2015 & $82.3^{c)}$ & $1,049.4^{c)}$ & $363,556.6^{c)}$ & $264.1^{c)}$ & NA \\
\hline Shui et al. [21], 2015 & $\begin{array}{c}103.8 \pm 59.4 \\
(11.0-247.0)\end{array}$ & $\begin{array}{c}1,197.3 \pm 744.2 \\
(114-4,000)\end{array}$ & $\begin{array}{l}454,016.2 \pm 282,200.6 \\
(43,228.8-1,516,800)\end{array}$ & $\begin{array}{c}253.1 \pm 109.3 \\
(100.4-708.2)\end{array}$ & $\begin{array}{c}66.2 \pm 48.6 \\
(4.2-373.3)\end{array}$ \\
\hline Zhou et al. [15], 2011 & $<180$ & $N A^{d)}$ & $N A^{d)}$ & $N A^{d)}$ & NA \\
\hline Wang et al. [16], 2009 & NA & $(468-3,413)$ & $(138,000-1,432,000)$ & NA & NA \\
\hline
\end{tabular}

Values are presented as mean \pm standard deviation (range), unless otherwise specified.

$\mathrm{NA}$, not available; $\mathrm{Cl}$, confidence interval; IQR, interquartile range.

${ }^{a}$ Treatment time (in minutes) was defined as the time from the first sonication to the last sonication, except in the study by Fan et al. [11], in which it was defined as the time from the first magnetic resonance localization scan to the last sonication. ${ }^{b}$ Sonication time (in seconds) was defined as the time of ablation when energy was delivered to the target. "Median value. ${ }^{\mathrm{d}}$ Mean treatment time between 1,132 and 1,820 seconds, mean total exposure energy between 384,637 and 765,571 J, and mean uterine volume between 213 and $253 \mathrm{~cm}^{3}$; varied depending on power output (290-420 W). 
Table 6. Summary of complications

\begin{tabular}{|c|c|c|c|c|c|}
\hline Complication & Fukunishi et al. [12] & Liu et al. [13] & Shui et al. [21] & Long et al. [14] & Zhou et al. [15] \\
\hline No. of patients & 20 & 230 & 350 & 51 & 77 \\
\hline Leg pain, prolonged ${ }^{\text {a) }}$ & $1(5)$ & - & - & - & - \\
\hline Sacrococcygeal or buttock pain, prolonged & $2(10)$ & - & - & $4(7.8)$ & $2(2.6)$ \\
\hline Abdominal pain, prolonged ${ }^{\mathrm{a})}$ & - & - & - & $1(1.9)$ & $1(1.3)$ \\
\hline Lower limb or perineal numbness & - & $2(0.8)$ & $1(0.3)$ & $9(17.6)$ & $3(3.9)$ \\
\hline Skin burn & - & $2^{\text {b) }}(0.8)$ & $3^{b)}(0.9)$ & - & $2^{b)}(2.6)$ \\
\hline Low grade fever & - & - & $1(0.3)$ & - & - \\
\hline Vaginal discharge or bleeding & - & $6(2.6)$ & $27(7.7)$ & $30(58.8)$ & $10(13.0)$ \\
\hline Hematuria & - & - & - & - & $2(2.6)$ \\
\hline Contact dermatitis to acoustic gel pad & $1(5)$ & - & - & - & - \\
\hline
\end{tabular}

sonication time, and the total exposure energy. The volume of the uteri and the adenomyotic lesions were also included when available for reference, as these values may have directly influenced the duration of treatment. Treatment time was generally defined as the time from the first sonication to last sonication, unless otherwise specified in the article, and sonication time referred to the time of ablation when energy was being delivered to the target.

\section{Complications}

Complications arising from HIFU in the treatment of adenomyosis were rare. Five of the 11 studies reported no adverse events or serious complications $[11,16,18-20]$. The complications reported in the other five studies are summarized in Table 6 . The most common complication was vaginal discharge or bleeding (range, $2.6 \%$ to $58.8 \%)$. Tissue burns, as the result of excessive power generated or accumulated in an incorrect target or in organs adjacent to the target, were uncommon. Skin burns, mostly of a mild degree, occurred in seven patients, and there were no reports of bowel or urinary bladder injury. The study carried out by Lee et al. [17] was excluded, as their safety data combined both fibroid and adenomyosis treatment.

\section{Discussion}

The management of uterine adenomyosis can be challenging, particularly in patients who wish to maintain their fertility [1]. While making treatment decisions, the age of the patients, severity of symptoms, desire for future fertility, and associated pelvic pathologies such as fibroids and endometriosis are important considerations. Various uterine-sparing interventions have been described in managing adenomyosis, including uterine artery embolization, myometrial or adenomyoma excision or reduction, or myometrial electrocoagulation $[1,2]$. However, insufficient properly designed trials have been conducted for adequate evidence to support one treatment over the other.

HIFU has been shown to be effective and safe in the treatment of uterine fibroids [6-8]. However, in treating adenomyosis, the effectiveness of HIFU is less well established and therefore, in our center, this treatment modality is still considered investigational. Adenomyosis can be a cause of significant morbidity, and further work is needed to explore more effective and safe therapies. The findings from this review seem encouraging, as HIFU appears to be effective in relieving, at least in part, the symptoms of adenomyosis, including menorrhagia and dysmenorrhea over a period of 1-2 years after treatment. This modality can be considered as an alternative option for women with adenomyosis who wish to preserve their uteri. However, as a fertility-sparing option, further evidence is certainly required to reassure clinicians and patients of the safety of this intervention if further pregnancy is desired.

This review includes studies of both MR-guided and US-guided HIFU. Although the principle of therapy is similar in both techniques, it is unclear whether one type of image guidance has advantages over the other. There is no doubt that the guiding images obtained during MR-guided therapy are much better than those obtained during US-guided therapy, especially during intraoperative mapping of the adenomyotic lesion, but whether this leads to better and safer treatment outcomes remains uncertain.

This review has limitations. The lack of primary data in most studies made it impossible to carry out comparative evaluations of certain important parameters, such as the degree of symptom relief, treatment time, and sonication time. Data combining both 
fibroid and adenomyosis treatment rendered a study unsuitable for evaluation [17]. The cost of each modality was not evaluated. The definitions of certain complications were not provided, so it was difficult to make comparisons between studies. Additionally, it is unfortunate that we were not able to include a recent large review consisting of 9,988 cases, of which 2,549 were adenomyosis, in this study, as no symptom outcome data were reported [6]. Nevertheless, despite these shortcomings, the availability of HIFU is indicative of the potential of advancements in adenomyosis treatment, and awareness of this treatment modality will allow physicians to provide optimal care to their patients.

\section{Conclusion}

HIFU appears to be effective and safe in the management of symptomatic adenomyosis, and can be considered as an alternative uterine-sparing option for women with this condition.

ORCID: Vincent Y. T. Cheung: http://orcid.org/0000-0002-3293-0697

\section{Conflict of Interest}

No potential conflict of interest relevant to this article was reported.

\section{References}

1. Struble J, Reid S, Bedaiwy MA. Adenomyosis: a clinical review of a challenging gynecologic condition. J Minim Invasive Gynecol 2016;23:164-185.

2. Grimbizis GF, Mikos T, Tarlatzis B. Uterus-sparing operative treatment for adenomyosis. Fertil Steril 2014;101:472-487.

3. Popovic M, Puchner S, Berzaczy D, Lammer J, Bucek RA. Uterine artery embolization for the treatment of adenomyosis: a review. J Vasc Interv Radiol 2011;22:901-909.

4. Mohan PP, Hamblin MH, Vogelzang RL. Uterine artery embolization and its effect on fertility. J Vasc Interv Radiol 2013;24:925-930.

5. Dong $X$, Yang Z. High-intensity focused ultrasound ablation of uterine localized adenomyosis. Curr Opin Obstet Gynecol 2010;22:326-330.

6. Chen J, Chen W, Zhang L, Li K, Peng S, He M, et al. Safety of ultrasound-guided ultrasound ablation for uterine fibroids and adenomyosis: a review of 9988 cases. Ultrason Sonochem 2015;27:671-676.

7. Cheung VY. Sonographically guided high-intensity focused ultrasound for the management of uterine fibroids. J Ultrasound Med 2013;32:1353-1358.

8. Cheung VY, Lam TP, Jenkins CR, Cheung GK, Chan SS, Choi WK. Ovarian reserve after ultrasound-guided high-intensity focused ultrasound for uterine fibroids: preliminary experience. J Obstet
Gynaecol Can 2016;38:357-361.

9. Lynn JG, Zwemer RL, Chick AJ, Miller AE. A new method for the generation and use of focused ultrasound in experimental biology. J Gen Physiol 1942;26:179-193.

10. Ng KK, Poon RT, Chan SC, Chok KS, Cheung TT, Tung H, et al. Highintensity focused ultrasound for hepatocellular carcinoma: a singlecenter experience. Ann Surg 2011;253:981-987.

11. Fan TY, Zhang L, Chen W, Liu Y, He M, Huang $X$, et al. Feasibility of MRI-guided high intensity focused ultrasound treatment for adenomyosis. Eur J Radiol 2012;81:3624-3630.

12. Fukunishi H, Funaki K, Sawada K, Yamaguchi K, Maeda T, Kaji Y. Early results of magnetic resonance-guided focused ultrasound surgery of adenomyosis: analysis of 20 cases. J Minim Invasive Gynecol 2008;15:571-579.

13. Liu X, Wang W, Wang Y, Wang Y, Li Q, Tang J. Clinical predictors of long-term success in ultrasound-guided high-intensity focused ultrasound ablation treatment for adenomyosis: a retrospective study. Medicine (Baltimore) 2016;95:e2443.

14. Long L, Chen J, Xiong Y, Zou M, Deng Y, Chen L, et al. Efficacy of high-intensity focused ultrasound ablation for adenomyosis therapy and sexual life quality. Int J Clin Exp Med 2015;8:11701-11707.

15. Zhou M, Chen JY, Tang LD, Chen WZ, Wang ZB. Ultrasound-guided high-intensity focused ultrasound ablation for adenomyosis: the clinical experience of a single center. Fertil Steril 2011;95:900-905.

16. Wang W, Wang Y, Tang J. Safety and efficacy of high intensity focused ultrasound ablation therapy for adenomyosis. Acad Radiol 2009;16:1416-1423.

17. Lee JS, Hong GY, Park BJ, Kim TE. Ultrasound-guided high-intensity focused ultrasound treatment for uterine fibroid and adenomyosis: a single center experience from the Republic of Korea. Ultrason Sonochem 2015;27:682-687.

18. Ferrari F, Arrigoni F, Miccoli A, Mascaretti S, Fascetti E, Mascaretti $G$, et al. Effectiveness of Magnetic Resonance-guided Focused Ultrasound Surgery (MRgFUS) in the uterine adenomyosis treatment: technical approach and MRI evaluation. Radiol Med 2016;121:153-161.

19. Polina L, Nyapathy V, Mishra A, Yellamanthili $H$, Vallabhaneni MP. Noninvasive treatment of focal adenomyosis with MR-guided focused ultrasound in two patients. Indian J Radiol Imaging 2012;22:93-97.

20. Kim KA, Yoon SW, Lee C, Seong SJ, Yoon BS, Park H. Short-term results of magnetic resonance imaging-guided focused ultrasound surgery for patients with adenomyosis: symptomatic relief and pain reduction. Fertil Steril 2011;95:1152-1155.

21. Shui L, Mao S, Wu Q, Huang G, Wang J, Zhang R, et al. Highintensity focused ultrasound (HIFU) for adenomyosis: two-year follow-up results. Ultrason Sonochem 2015;27:677-681.

22. Yoon SW, Kim KA, Cha SH, Kim YM, Lee C, Na YJ, et al. Successful use of magnetic resonance-guided focused ultrasound surgery to 
relieve symptoms in a patient with symptomatic focal adenomyosis. Fertil Steril 2008;90:2018.e2013-2015.

23. Zhang $X$, Li K, Xie B, He M, He J, Zhang L. Effective ablation therapy of adenomyosis with ultrasound-guided high-intensity focused ultrasound. Int I Gynaecol Obstet 2014;124:207-211.

24. Spies JB, Coyne K, Guaou Guaou N, Boyle D, Skyrnarz-Murphy K,
Gonzalves SM. The UFS-QOL, a new disease-specific symptom and health-related quality of life questionnaire for leiomyomata. Obstet Gynecol 2002;99:290-300.

25. Sharp NC, Cronin N, Feldberg I, Evans M, Hodgson D, Ellis S. Microwaves for menorrhagia: a new fast technique for endometrial ablation. Lancet 1995;346:1003-1004. 